\title{
Cytotoxic effects of acrylonitrile on human umbilical cord mesenchymal stem cells in vitro
}

\author{
XIAOCHUN SUN, MIN SUN, YAN XIE, WEI ZHAI, WEI ZHU, RUI MA, RONGZHU LU and WENRONG XU
}

School of Medical Science and Laboratory Medicine, Jiangsu University, Zhenjiang, Jiangsu 212013, P.R. China

Received May 9, 2013; Accepted November 5, 2013

DOI: $10.3892 / \mathrm{mmr} .2013 .1802$

\begin{abstract}
The effects of acrylonitrile (ACN) on human umbilical cord mesenchymal stem cells (hUC-MSCs) remain unknown. The proliferation, differentiation, clonogenicity and apoptosis effects of ACN and/or N-acetyl-L-cysteine (NAC) on hUC-MSCs were investigated. The results showed that although $\mathrm{ACN}$ at a concentration of $0.1 \mu \mathrm{g} / \mathrm{ml}$ did not affect proliferation or the morphology of hUC-MSCs compared with the control, osteogenic differentiation and the positive rate of alkaline phosphatase staining in the experimental group were significantly lower compared with the control $(\mathrm{P}<0.01)$. All of the effects of ACN were counteracted using NAC, a typical antioxidant. Using a flow cytometry assay, it was observed that ACN induced apoptosis in hUC-MSCs. The results indicated that the toxic effect produced by ACN on hUC-MSCs is based on a redox mechanism.
\end{abstract}

\section{Introduction}

Acrylonitrile (ACN), an industrial nitrile, is a widely used intermediate in the manufacturing of acrylic fibers, plastics, synthetic rubbers, adhesives and pesticides. Potential human exposure to ACN may occur during its production and through the use of ACN-containing products. In clinical applications, $\mathrm{ACN}$ is used in the synthesis of biomaterials, including high permeable dialysis tubing (1) and artificial membranes to encapsulate Langerhans islet implants (2). Furthermore, ACN has been detected in drinking water, cigarette smoke, food and occupational environments (3-5). An increase in cancers, mainly of the lung, gastrointestinal and hemopoietic-lymphatic system, in workers occupationally exposed to ACN has been reported (6). The International Agency for Research on Cancer (IARC) classified ACN as 'possibly carcinogenic to humans' (7).

Correspondence to: Professor Xiaochun Sun, School of Medical Science and Laboratory Medicine, Jiangsu University, 301 Xuefu Road, Zhenjiang, Jiangsu 212001, P.R. China

E-mail: xiaochun@ujs.edu.cn; E-mail: sunxiaochun518@163.com

Key words: human umbilical cord mesenchymal stem cells, acrylonitrile, $\mathrm{N}$-acetyl-L-cysteine, cytotoxic
ACN is acutely toxic to humans. While the America National Institute for Occupational Safety and Health recommended permissible exposure level to ACN is relatively low (1 ppm), high-level exposure may be reached via skin contact in the case of accidental exposure (8). Studies performed on animals indicated that ACN exhibits mutagenic, embryotoxic (9), carcinogenic, immunotoxic (10) and hematotoxicity (11) effects. An earlier study demonstrated that blood retains high levels of ACN (12) and indicated a metabolic incorporation and macromolecular interaction of $\mathrm{ACN}$ in the liver, spleen, bone marrow, lung and adipose tissues of rats (13). A previous study also indicated that $\mathrm{ACN}$ may interfere with their proliferative activity and with the complex regulation pathways, by modulation of gene and protein expression in hemopoietic cells (14).

$\mathrm{N}$-acetyl-L-cysteine (NAC) is the acetylated precursor of the amino acid L-cysteine and glutathione (GSH). The biological activity of NAC is attributed to its protection against oxidative and metabolic processes (15). NAC is a powerful nucleophile capable of scavenging free radicals, stimulating GSH synthesis and enhancing glutathione-S-transferase activity. It has been observed that NAC may prevent $\mathrm{ACN}$-induced damage in glial cells (16).

Stem cells have been observed to be significant in predicting toxicity by working with in vitro systems (17) and mesenchymal stem cells (MSCs) have attracted more attention due to its multipotency to differentiate into a variety of cell types of mesodermal lineage $(18,19)$. In addition, in clinical practice, human umbilical cord MSCs (hUC-MSCs) may be harvested in a safer and more non-invasive manner than bone marrow (BM) and have emerged as a possible alternative cell source to BM-MSCs with less ethical controversy than embryonic stem cells.

There is a lack of information with regard to the potential toxicity of ACN in MSCs. Therefore, the objective of the present study was to investigate the potential cytotoxic effects, as well as the underlying mechanisms of toxicity, induced by $\mathrm{ACN}$ in hUC-MSCs.

\section{Materials and methods}

Isolation of human umbilical cord cells. Fresh human umbilical cords were obtained from the Fourth Hospital of Zhenjiang and maintained in sterile conditions at $4^{\circ} \mathrm{C}$. The surface of each cord was rinsed with phosphate-buffered saline (PBS) to remove as much blood as possible and the cord 
was sliced into 3-5 $\mathrm{cm}$-long sections using sharp, sterile scissors. Blood vessels were removed from each piece of cord then the rest of the tissue was sliced into small fragments $\sim 1 \mathrm{~mm}^{3}$. The fragments were seeded onto the surface of a culture dish (with a diameter of $3 \mathrm{~cm}$ ) with low glucose Dulbecco's modified Eagle's medium (L-DMEM; Gibco-BRL, Carlsbad, CA, USA) supplemented with $10 \%(\mathrm{v} / \mathrm{v})$ fetal bovine serum (FBS), penicillin $(100 \mathrm{U} / \mathrm{l})$ and streptomycin $(100 \mu \mathrm{g} / \mathrm{ml})$ at $37^{\circ} \mathrm{C}$ in $5 \% \mathrm{CO}_{2}-95 \%$ air atmosphere for two weeks. Following two weeks incubation, the explants were removed leaving the cells that had adhered to the plate. When cells grew to $70 \%$ confluency, they were harvested and plated onto a $25-\mathrm{cm}^{2}$ culture flask. The experimental procedure was approved by Jiangsu University Ethics Committee (Zhenjiang, China) and written informed consent was obtained from the patients.

Cell proliferation and survival assay. The viability and proliferation of cells were determined by 3-(4,5-dimethylthiazol-2-yl)-2,5-diphenyltetrazolium bromide (MTT) assay. Briefly, the cells were plated in 96-well plates and once cells reached $70-80 \%$ confluency, the medium was removed and incubated with various concentrations of $\mathrm{ACN}$-dissolved serum-free culture medium for 12 and $24 \mathrm{~h}$, respectively. MTT $(20 \mu \mathrm{l})$ was added to each well in the final $4 \mathrm{~h}$. The supernatant was discarded and $150 \mu \mathrm{l}$ dimethyl sulfoxide (DMSO) was added to each well. Following uniform oscillation for $10 \mathrm{~min}$ to fully dissolve the crystals, the absorption values were examined using a microplate reader (Biotek, Winooski, VT, USA) with a wavelength of $570 \mathrm{~nm}$.

The hUC-MSCs were plated in 96-well plates and once $70-80 \%$ cell confluency was reached, the medium was removed with specific concentrations of NAC serum-free culture medium. After $30 \mathrm{~min}, 0.1 \mu \mathrm{g} / \mathrm{ml}$ ACN was added to each well. Following $24 \mathrm{~h}$ incubation, the viability of the cultured hUM-MSCs was determined by MTT.

Osteogenic differentiation. The multipotent differentiation of the hUC-MSCs was analyzed for osteogenic ability. The cells were inoculated into 24 -well plate at 3,000 cells/well in DMEM supplemented with 10\% FBS and incubated in a modified version of differentiation medium (containing $4 \mathrm{mg} / \mathrm{l}$ basic fibroblast growth factor). The cells were treated for two weeks with osteogenic induction medium $(0.1 \mu \mathrm{M}$ dexamethasone, $10 \mathrm{mM}$-glycerophosphate, $4 \mathrm{mg} / \mathrm{l}$ basic fibroblast growth factor and $50 \mu \mathrm{g} / \mathrm{ml}$ ascorbic acid).

Quantitative polymerase chain reaction ( $q P C R$ ) assay. Cell lysis and total RNA extraction from control and treated cells treated with ACN using TRIzol Reagent (Invitrogen Life Technologies, Carlsbad, CA, USA) was performed according to the reverse transcription kit instructions (Fermentas, Waltham, MA, USA). The cDNA samples were subjected to PCR using specific primers. The primers were designed and synthesized by Invitrogen Life Technologies according to the serial number from GenBank (Table I). The reaction was started at $94^{\circ} \mathrm{C}$ for $5 \mathrm{~min}$, denaturation at $94^{\circ} \mathrm{C}$ for $30 \mathrm{sec}$, annealing at $55-70^{\circ} \mathrm{C}$ for $30 \mathrm{sec}$ and extension for $30 \mathrm{sec}$ at $72^{\circ} \mathrm{C}$ followed by 30 cycles and a final polymerization at $72^{\circ} \mathrm{C}$ for 10 min. $\beta$-actin mRNA was used as an internal control. The products were checked by electrophoresis on a $1.5 \%$ agarose gel, with ethidium bromide staining and analyzed using the Gel Image Analysis System (Syngene, Cambridge, UK).

Cytochemical staining. Following hUC-MSC differentiation, osteogenic characteristics were confirmed through alkaline phosphatase (ALP) expression by cytochemical staining. Cells were fixed with $4 \%$ paraformaldehyde. The ALP staining kit (Sun Biotech Co., Ltd., Shanghai, China) was used according to the manufacturer's instructions.

Cell cycle assay. hUC-MSCs were treated with ACN and NAC for $24 \mathrm{~h}$. Cells were harvested and washed twice with PBS and stained with propidium iodide (PI; Sigma-Aldrich, St. Louis, MO, USA) for $30 \mathrm{~min}$ in dark conditions. The stained cells were analyzed by flow cytometry (FACSCalibur; BD Biosciences, San Diego, CA, USA).

Apoptosis assay. hUC-MSCs were treated with ACN and NAC for $24 \mathrm{~h}$. Following treatment, the cells were trypsinized with $0.25 \%$ trypsin-EDTA, washed twice with PBS and stained according to the recommendation of the manufacturer with propidium iodide (PI) and Annexin V-fluorescein isothiocyanate (FITC). The stained cells were analyzed by flow cytometry (FACSCalibur).

\section{Results}

Cell morphology and immunological phenotype. hUC-MSCs were observed following an initial three days of primary culture. The cells adhered to plastic surfaces and presented as a small population of single cells with spindle shape. After 7-10 days, the cells appeared as long spindle-shaped fibroblastic cells (Fig. 1). Following re-plating, the fibroblast-like cells appeared polygonal or spindly, with a long process and were considered normal, on the basis of typical morphology.

Effect of ACN on cell proliferation. To investigate the cytotoxicity of ACN on hUC-MSC proliferation, cells were exposed to specific concentrations of $\mathrm{ACN}(0,0.05,0.1,0.2,1,10,20$ and $50 \mu \mathrm{g} / \mathrm{ml}$ ) for 12 and $24 \mathrm{~h}$. The MTT assay was used to determine ACN-induced toxicity in hUC-MSC. Following ACN treatment, the activation of hUC-MSC was markedly decreased in a dose- and time-dependent manner (Fig. 2), indicating that $\mathrm{ACN}$ is cytotoxic in hUC-MSC.

Effect of ACN on osteogenic differentiation. To examine the potential effect of ACN on hUC-MSC to differentiate into osteocytes, the cells were cultured in osteogenic medium. Following 14 days, ALP stain and qPCR was applied for characterization of the osteogenic differentiation. Compared with the control group, the positive rate of ALP was reduced in the $\mathrm{ACN}$ group (Fig. $3 \mathrm{~A}$ and $\mathrm{B} ; \mathrm{P}<0.01$ ). $\mathrm{ACN}$ induced a statistically significant difference in the expression of the $A L P$ gene (Fig. 3C), which is considered to be a marker for osteocytes. These results indicated that $\mathrm{ACN}$ inhibits the osteogenic differentiation of MSCs.

Effect of ACN on specific genes of hematogenesis. qPCR was applied for characterization of expression of cytokines in hUC-MSCs, which are important components of the 
Table I. Specific primers for control and target genes.

\begin{tabular}{|c|c|c|c|}
\hline Gene & Primers sequence, $5^{\prime}$ to $3^{\prime}$ & Size (bp) & Annealing, ${ }^{\circ} \mathrm{C}$ \\
\hline Flt3-ligand-F & CTGGAGCCCAACAACCTATC & 353 & 60.0 \\
\hline Flt3-ligand-R & TCTGGACGAAGCGAAGACA & & \\
\hline SCF-F & TGGATAAGCGAGATGGTA & 189 & 54.0 \\
\hline SCF-R & TTCTGGGCTCTTGAATGA & & \\
\hline VEGF-F & CСТTGСТСТАССТССАС & 280 & 61.0 \\
\hline VEGF-R & ATCTGCATCCTGTTGGA & & \\
\hline ALP-F & AGCTTCAAACCGAGATACAA & 220 & 56.5 \\
\hline ALP-R & ATTCTGCCTCCTTCCACC & & \\
\hline$\beta$-actin-F & CACGAAACTACCTTCAACTC & 256 & 56.0 \\
\hline$\beta$-actin-R & CATACTCCTGCTTGCTGATC & & \\
\hline
\end{tabular}

Flt3, Fms-like tyrosine kinase 3; SCF, stem cell factor; VEGF, vascular endothelial growth factor; ALP, alkaline phosphatase; F, forward; $\mathrm{R}$, reverse.

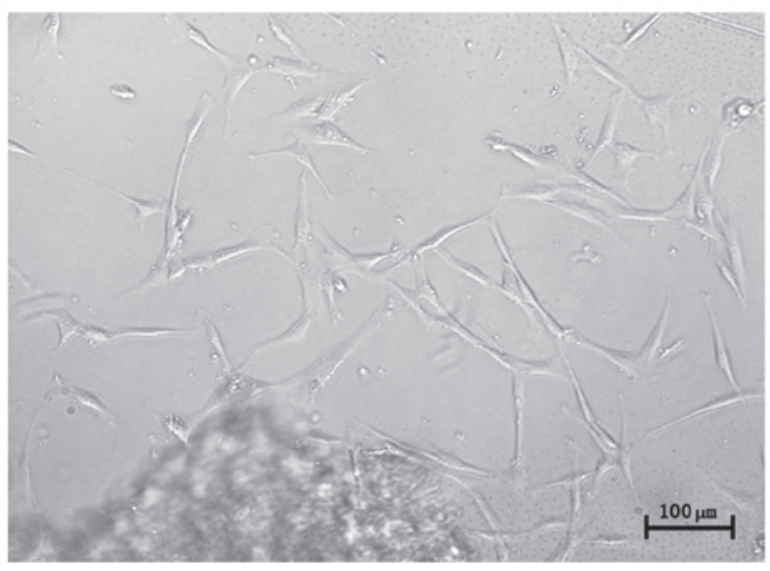

Figure 1. Morphology of MSCs from hUC-MSCs. hUC-MSCs were long, spindle-shaped and fibroblastic in appearance following 10 days of primary culture. Magnification, x100. MSCs, mesenchymal stem cells; hUC-MSCs, human umbilical cord MSCs.

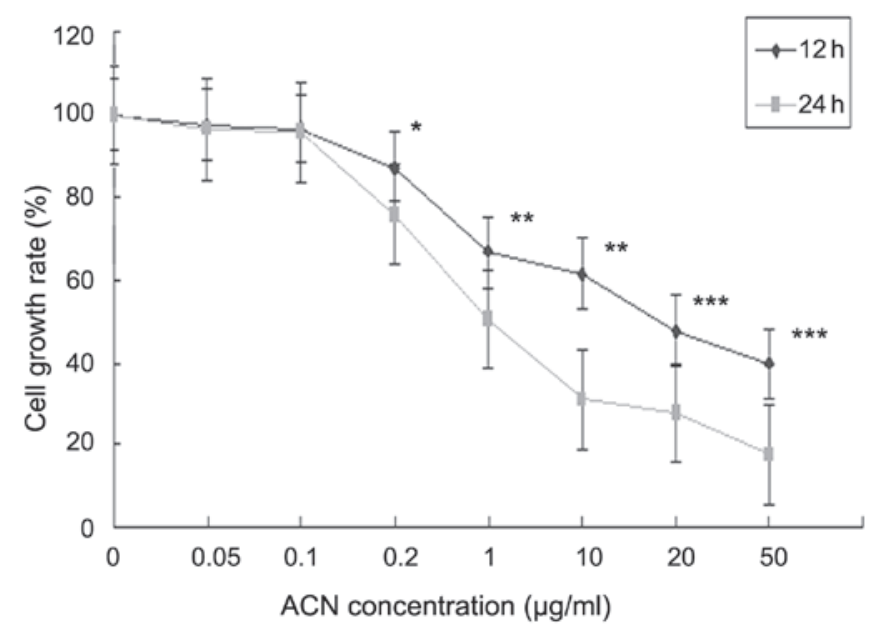

Figure 2. Effects of cell viability of ACN on hUC-MSCs. Cells were treated with $0.05,0.1,0.2,1,10,20$ and $50 \mu \mathrm{g} / \mathrm{ml} \mathrm{ACN}$ for 12 or $24 \mathrm{~h}$ and cell viability was determined by an MTT assay. ${ }^{*} \mathrm{P}<0.05,{ }^{* *} \mathrm{P}<0.01$ and ${ }^{* * *} \mathrm{P}<0.001$, vs. the control. hUC-MSCs, human umbilical cord mesenchymal stem cells; ACN, acrylonitrile; MTT, 3-(4,5-dimethylthiazol-2-yl-)-2,5-diphenyl tetrazolium bromide. hematopoietic microenvironment, in addition to MSCs. There were dose-dependent decreases in the expression of cytokine vascular endothelial growth factor (VEGF), stem cell factor (SCF) and Fms-like tyrosine kinase 3 (Flt3) showed with treatment of ACN (Fig. 4). These results indicated that ACN may injure the hematopoietic system by inhibiting the hematopoiesis-supportive function of MSCs.

NAC attenuates ACN-induced cytotoxicity. Since ACN is capable of inducing oxidative stress, it was investigated whether ACN was capable of inducing oxidative stress in MSCs. Cells were pretreated with an antioxidant, NAC, at different concentrations followed by ACN treatment and cytoactivity was determined. The MTT assay showed that treatment with NAC resulted in a dose-dependent increase in cytoactivity at specific concentrations. The optimal effect was observed at $3 \mathrm{mM}$, at which point a further increase in NAC did not show any additional benefit (Fig. 5A). Thus, $3 \mathrm{mM}$ was selected as the concentration of NAC for further studies.

The protection of NAC on ACN-treated cells was further investigated. They all showed significant differences in ACN-treated only groups of concentration $0.05,0.1$, and $0.2 \mu \mathrm{g} / \mathrm{ml}$, as compared with the corresponding ACN+NAC groups (Fig 5).

Effect of ACN on cell cycle. Flow cytometry was used to determine whether the inhibitory effect of ACN on MSC proliferation was mediated, at least in part, by affecting cell cycle progression. The results demonstrated that pretreatment with NAC attenuated ACN-induced cell cycle arrest at the G2/M phase and suggested that ACN suppresses cell proliferation by controlling the $\mathrm{G} 2 / \mathrm{M}$ checkpoint and inducing a specific block in cell cycle progression (Fig. 6).

ACN induces cellular apoptosis. To further study the effect of ACN on hUC-MSC apoptosis, cells were stained with Annexin V/FITC and PI and subsequently analyzed by flow cytometry. Flow cytometric analysis showed that the percentage of hUC-MSCs undergoing apoptosis following ACN-treatment were significantly higher compared with that of the control 
A

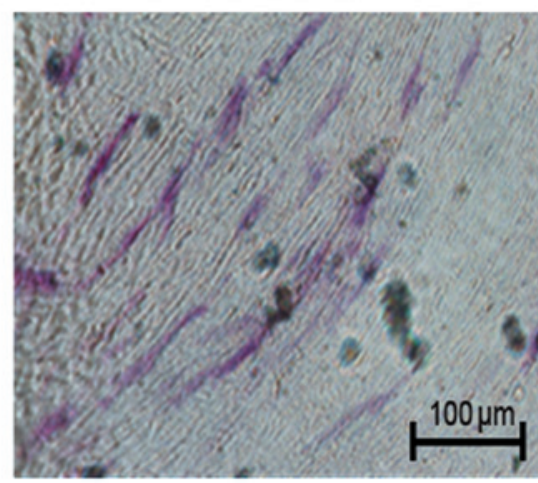

Control $(\times 100)$

B

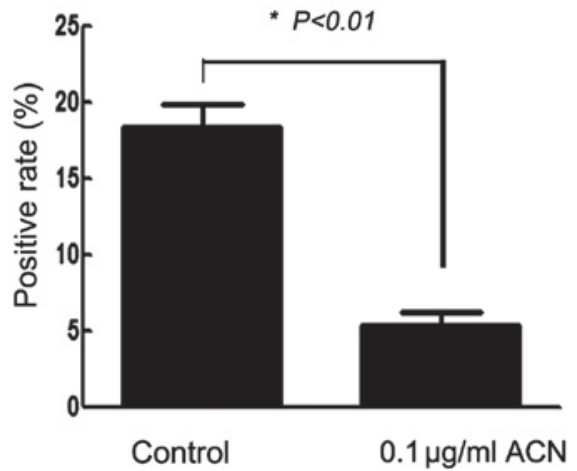

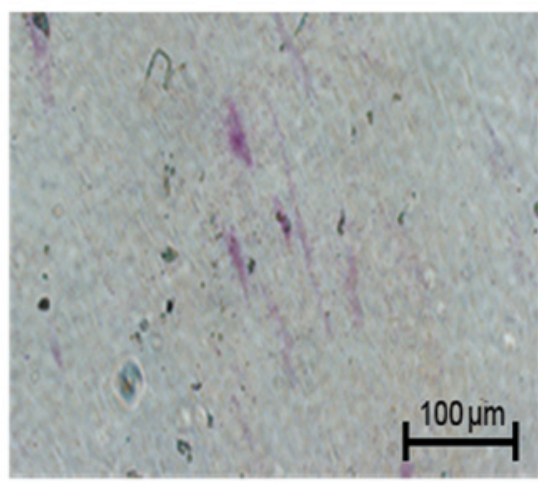

$0.1 \mu \mathrm{g} / \mathrm{ml} \mathrm{ACN}(\times 100)$

C

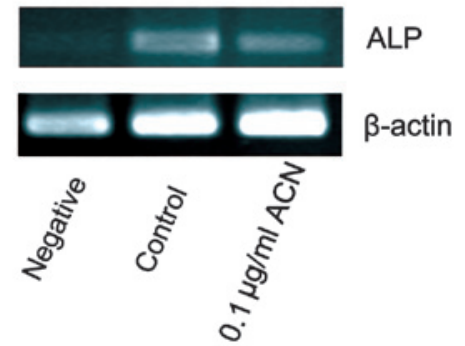

Figure 3. ACN inhibits osteogenic differentiation of hUC-MSCs. (A) Cells were induced towards osteogenic differentiation, in the presence or not in the presence of $100 \mu \mathrm{g} / \mathrm{ml} \mathrm{ACN}$ for 14 days. The presence of alkaline phosphatase positive was assessed by microscopic observation of histochemical staining. (B) Graphical representation of positive rate of ALP staining in cells. "P<0.01, vs. the control. (C) ALP mRNA levels were estimated by qPCR assays. ACN, acrylonitrile; hUC-MSCs, human umbilical cord mesenchymal stem cells; ALP, alkaline phosphatase; qPCR, quantitative polymerase chain reaction.

FIt3

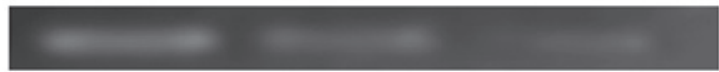

SCF

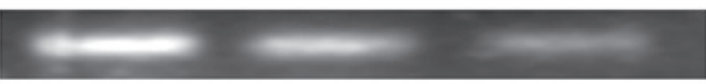

VEGF

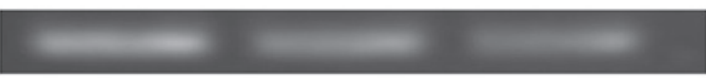

$\beta$-actin

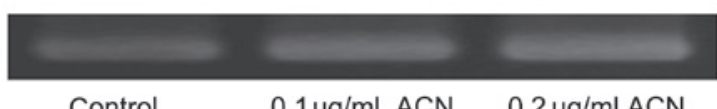

Control

$0.1 \mu \mathrm{g} / \mathrm{ml} \mathrm{ACN}$

$0.2 \mu \mathrm{g} / \mathrm{ml} \mathrm{ACN}$

Figure 4. Relative quantification by qPCR of gene expression levels of cytokine following exposure to ACN. Cells were treated with 100 or $200 \mu \mathrm{g} / \mathrm{ml}$ ACN for $12 \mathrm{~h}$. ACN, acrylonitrile; qPCR, quantitative polymerase chain reaction.

cells $(\mathrm{P}<0.01)$ and the NAC pretreatment group $(\mathrm{P}<0.05)$, thus implying that ACN may induce the apoptosis of hUC-MSCs and may be counteracted with the use of NAC (Fig. 7).

\section{Discussion}

There is evidence that the metabolism of ACN to epoxide intermediate 2-cyanoethylene oxide, to form adducts with
DNA, may contribute to the toxicity and carcinogenicity of ACN.

$\mathrm{BM}$ is the site for hematopoiesis to occur, as well as in the cord blood, where there are MSCs that are capable of differentiating into multiple cell types, including adipocytes, chondrocytes, osteocytes and cardiomyocytes (20). The present study provides evidence that ACN suppresses cytoactivity, differentiation and causes apoptosis in hUM-MSCs. Doses for the current study were selected on the basis of the results of a $24 \mathrm{~h}$ in vitro study. ACN $(0.1 \mu \mathrm{g} / \mathrm{ml})$ was observed to affect the proliferation and morphology of MSCs.

The present study demonstrated that ACN disturbed the balance of cell proliferation and induced cell apoptosis, as well as the potential for differentiation. Following osteogenic induction, qPCR analysis of the gene expression of an early marker of cells oriented towards osteogenic production demonstrated downregulation of ALP (21) following ACN treatment, which is an intracellular enzyme required for mineralization. Histological staining supported the qPCR data by demonstrating the presence of ALP positive cells. Therefore, in the present study, ACN exhibited down-regulation of the osteogenic capacity of MSCs.

MSCs produce a number of cytokines and extracellular matrix proteins and express cell adhesion molecules, which are critical for hematopoiesis (22), thus, the ACN effect on the hematopoiesis by MSCs was investigated. In the present study, 

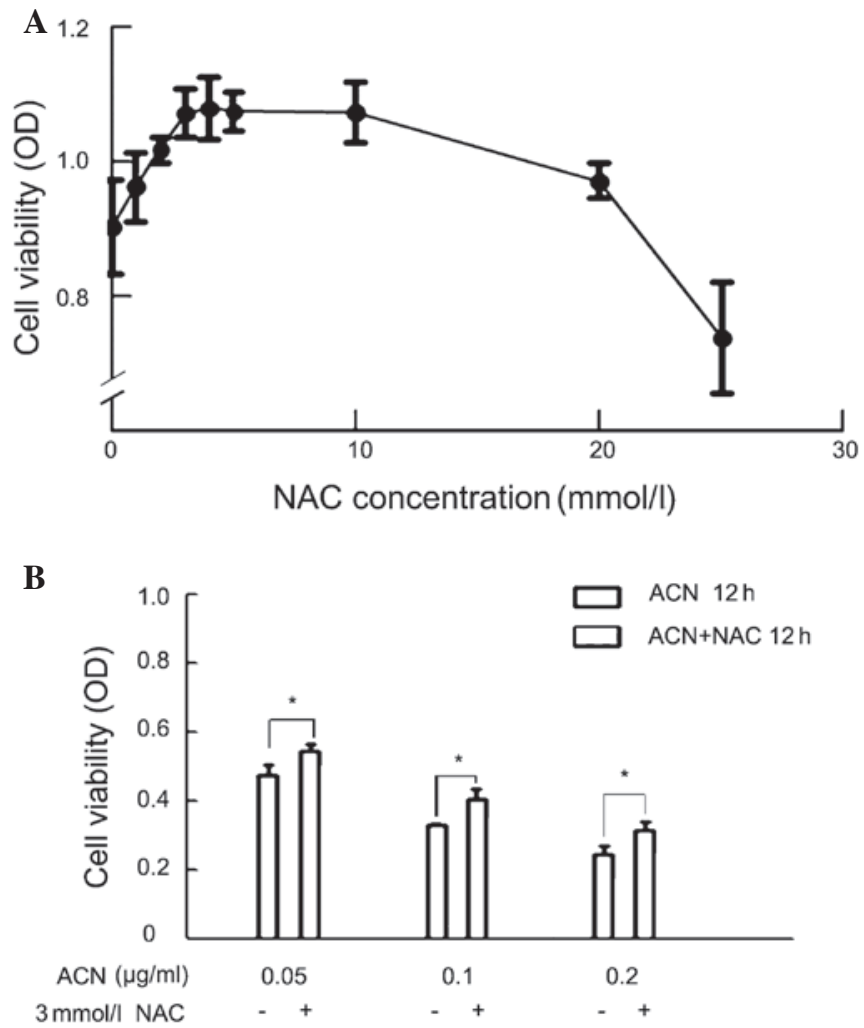

Figure 5. Effects of cell viability of NAC on hUC-MSCs. (A) hUC-MSCs were pretreated with different concentrations of NAC (1-25 mM). (B) Attenuation of ACN-induced cytotoxicity by pretreatment with NAC. Cell viability was determined by MTT. "P<0.05, vs. ACN 12 h. NAC, N-acetyl-L-cysteine; hUC-MSCs, human umbilical cord mesenchymal stem cells; ACN, acrylonitrile; MTT, 3-(4,5-dimethylthiazol-2-yl-)-2,5-diphenyl tetrazolium bromide; OD, optical density.
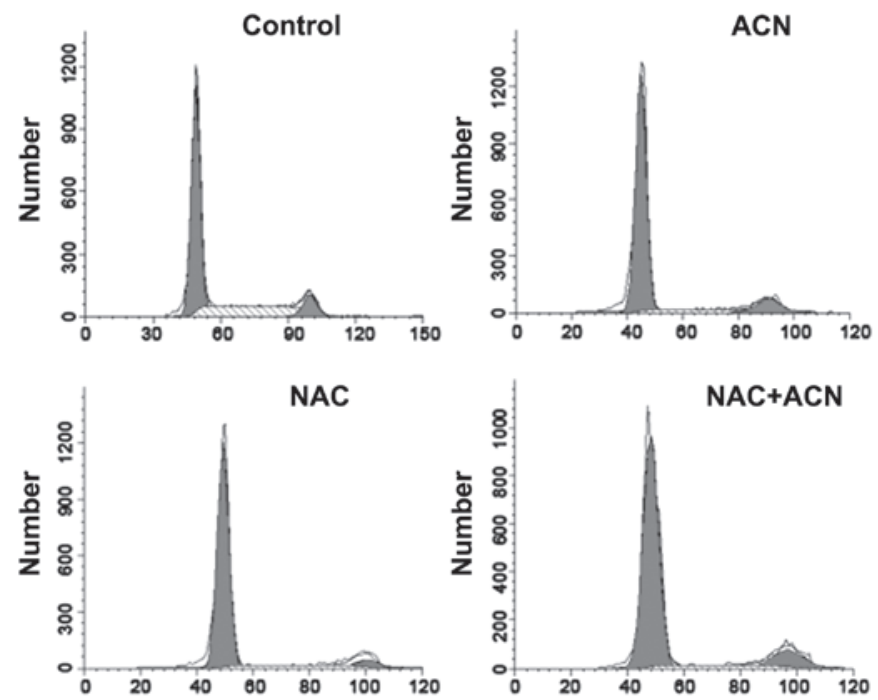

Figure 6. Effect of ACN on the cell-cycle of hUC-MSCs. The DNA content analysis indicated the percentage of cells in the G2/M phase was higher in ACN-treated compared with that of the control. hUC-MSCs, human umbilical cord mesenchymal stem cells; ACN, acrylonitrile.

qPCR experiments determined mRNA marker expression for a number of the hematopoietic cytokines. VEGF, Flt3 and SCF were downregulated following exposure to ACN. These results
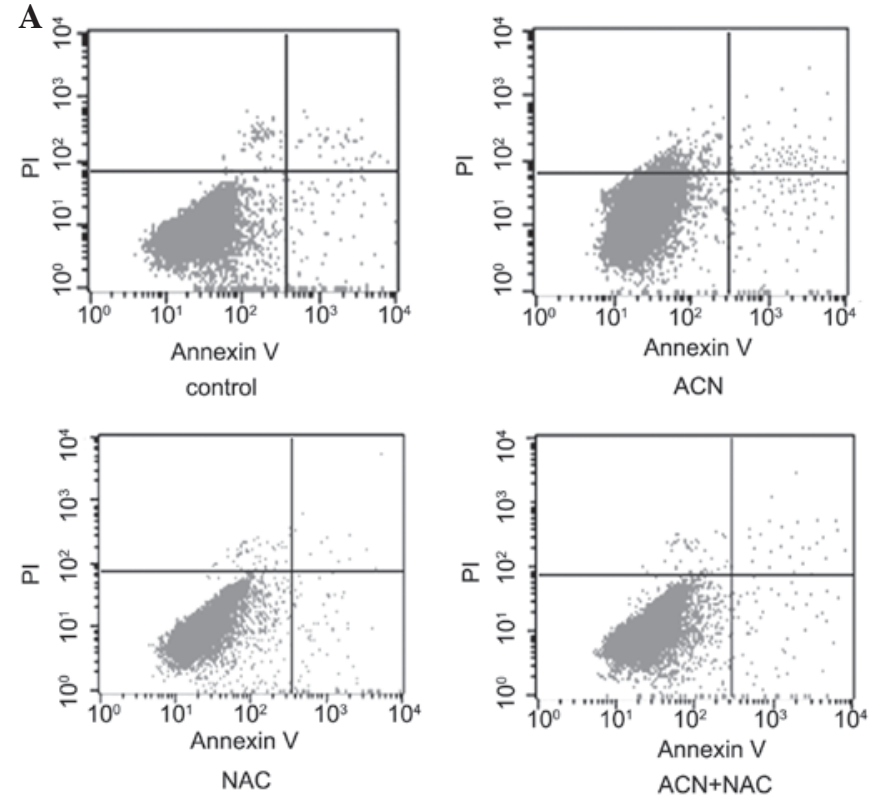

B

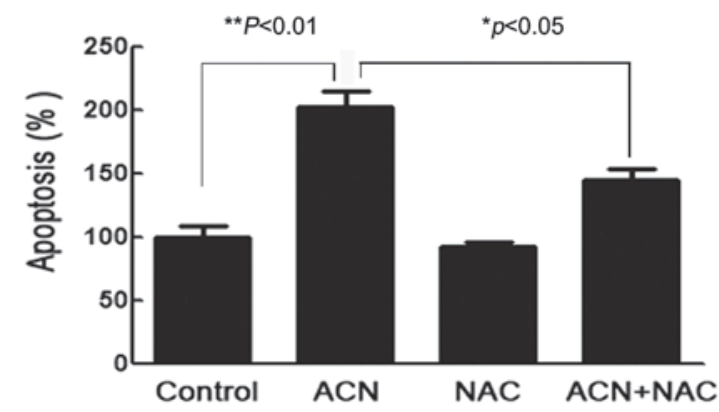

Figure 7. Effect of ACN-induced apoptosis in hUC-MSCs. (A) Representative scattergrams from fluorescence-activated cell sorting profile represents Annexin V-fluorescein isothiocyanate staining on the $\mathrm{x}$-axis and PI on the $y$-axis. (B) Graphical representation of apoptotic in cells treated with or without ACN. " $\mathrm{P}<0.05$, vs. ACN-treated cells and ${ }^{* *} \mathrm{P}<0.01$, vs. the control. hUC-MSCs, human umbilical cord mesenchymal stem cells; ACN, acrylonitrile; PI, propidium iodide.

indicate that ACN may destroy the hematopoietic microenvironment. It has been reported that $\mathrm{ACN}$ was extremely reactive with rat tissue proteins in vivo (23). Blood was the most reactive tissue studied and hemoglobin was the most reactive protein in blood. MSCs exhibit multilineage differentiation potential and are capable of generating progenitors with restricted developmental potential (24).

In addition, ACN inhibited cell proliferation with a smaller increase in apoptosis. A previous study demonstrated that ACN induces apoptosis (25) and further identified that the mechanism of ACN-induced cell death in hUC-MSCs is through induction of apoptosis involved in the generation of oxidative stress. ACN was observed to cause apoptosis in hUC-MSCs, as was observed by flow cytometry. A number of studies have been published stating that the antioxidant, NAC, protects the rat neurocyte from ACN toxicity, suggesting the involvement of oxidative stress in ACN toxicity. Using NAC as a potential protective agent may induce the apoptosis of hUC-MSCs. Oxidative stress is important in the initiation of apoptosis.

Despite a considerable amount of research, the mechanism directly responsible for ACN toxicity remains unclear. Three 
distinct pathways have been proposed: i) Reactive oxygen species (ROS) generated as by-products of ACN metabolism via cytochrome P450 2E1 oxidation (26). ii) Liberation of cyanide from ACN metabolism. Cyanide is a potent generator of ROS production as well as an inhibitor of the activities of several antioxidant enzymes $(27,28)$. iii) The process of ACN conjugation with GSH, results in a rapid depletion of GSH and an overall decrease in cellular antioxidant contents. A number of studies have indicated the role of stress in the toxicity of ACN. Esmat et al (29) reported that NAC protects glial cells from ACN toxicity, suggesting the attribution of oxidative stress in ACN toxicity.

In conclusion, $\mathrm{ACN}$ may inhibit the proliferation, differentiation and cytokine gene expression of hUC-MSCs and the present study supports the hypothesis that ACN causes apoptosis in hUC-MSCs through a mechanism involving the generation of oxidative stress. We, therefore, require further investigation of the antagonism of antioxidant in these ACN effects.

\section{Acknowledgements}

This study was supported by grants from the National Natural Science Foundation of China (grant no. 30840053) and the Foundation of the Jiangsu University for Senior talented Scholars (grant no. 11JDG0089).

\section{References}

1. Ward RA, Schaefer RM, Falkenhagen D, et al: Biocompatibility of a new high-permeability modified cellulose membrane for haemodialysis. Nephrol Dial Transplant 8: 47-53, 1993.

2. Kessler L, Pinget M, Aprahamian M, Dejardin P and Damgé C: In vitro and in vivo studies of the properties of an artificial membrane for pancreatic islet encapsulation. Horm Metab Res 23: 312-317, 1991

3. Léonard A, Gerber GB, Stecca C, et al: Mutagenicity, carcinogenicity, and teratogenicity of acrylonitrile. Mutat Res 436 263-283, 1999.

4. Miller SL, Branoff S and Nazaroff WW: Exposure to toxic air contaminants in environmental tobacco smoke: an assessment for California based on personal monitoring data. J Expo Anal Environ Epidemiol 8: 287-311, 1998.

5. Rubio PA: Use of adhesive tape for primary closure of surgical skin wounds. Int Surg 75: 189-190, 1990.

6. Ward CE and Starr TB: Comparison of cancer risks projected from animal bioassays to epidemiologic studies of acrylonitrile-exposed workers. Regul Toxicol Pharmacol 18: 214-232, 1993.

7. Fechter LD, Klis SF, Shirwany NA, et al: Acrylonitrile produces transient cochlear function loss and potentiates permanent noiseinduced hearing loss. Toxicol Sci 75: 117-123, 2003.

8. Pouyatos B, Gearhart CA, Nelson-Miller A, et al: vulnerability of the cochlear Basal turn to acrylonitrile and noise. J Toxicol 2009 908596, 2009.

9. Parent RA and Casto BC: Effect of acrylonitrile on primary Syrian golden hamster embryo cells in culture: transformation and DNA fragmentation. J Natl Cancer Inst 62: 1025-1029, 1979.
10. Hamdy NM, Al-Abbasi FA, Alghamdi HA, Tolba MF, Esmat A and Abdel-Naim AB: Role of neutrophils in acrylonitrile-induced gastric mucosal damage. Toxicol Lett 208: 108-114, 2012.

11. Ghanayem BI, Elwell MR and Eldridge SR: Effects of the carcinogen, acrylonitrile, on forestomach cell proliferation and apoptosis in the rat: comparison with methacrylonitrile. Carcinogenesis 18: 675-680, 1997.

12. Ahmed AE, Farooqui MY, Upreti RK and El-Shabrawy O: Comparative toxicokinetics of 2,3-14C- and 1-14C-acrylonitrile in the rat. J Appl Toxicol 3: 39-47, 1983.

13. Jacob S and Ahmed AE: Effect of route of administration on the disposition of acrylonitrile: quantitative whole-body autoradiographic study in rats. Pharmacol Res 48: 479-488, 2003.

14. Diodovich C, Malerba I, Ferrario D, et al: Gene and protein expressions in human cord blood cells after exposure to acrylonitrile. J Biochem Mol Toxicol 19: 204-212, 2005.

15. Sjödin K, Nilsson E, Hallberg A and Tunek A: Metabolism of N-acetyl-L-cysteine. Some structural requirements for the deacetylation and consequences for the oral bioavailability. Biochem Pharmacol 38: 3981-3985, 1989.

16. Carrera MP, Antolín I, Martin V, et al: Antioxidants do not prevent acrylonitrile-induced toxicity. Toxicol Lett 169: 236-244, 2007.

17. Chapin RE and Stedman DB: Endless possibilities: stem cells and the vision for toxicology testing in the 21st century. Toxicol Sci 112: 17-22, 2009

18. Nagaya N, Fujii T, Iwase T, et al: Intravenous administration of mesenchymal stem cells improves cardiac function in rats with acute myocardial infarction through angiogenesis and myogenesis. Am J Physiol Heart Circ Physiol 287: H2670-H2676, 2004.

19. Prockop DJ: Marrow stromal cells as stem cells for nonhematopoietic tissues. Science 276: 71-74, 1997.

20. Orbay H, Tobita M and Mizuno H: Mesenchymal stem cells isolated from adipose and other tissues: basic biological properties and clinical applications. Stem Cells Int 2012: 461718, 2012.

21. Sugawara Y, Suzuki K, Koshikawa M, Ando M and Iida J: Necessity of enzymatic activity of alkaline phosphatase for mineralization of osteoblastic cells. Jpn J Pharmacol 88: 262-269, 2002.

22. Weissman IL: Stem cells: units of development, units of regeneration, and units in evolution. Cell 100: 157-168, 2000.

23. Campian EC and Benz FW: The acute lethality of acrylonitrile is not due to brain metabolic arrest. Toxicology 253: 104-109, 2008

24. Fibbe WE: Mesenchymal stem cells. A potential source for skeletal repair. Ann Rheum Dis 61 (Suppl 2): ii29-ii31, 2002.

25. Watcharasit P, Suntararuks S, Visitnonthachai D, Thiantanawat A and Satayavivad J: Acrylonitrile induced apoptosis via oxidative stress in neuroblastoma SH-SY5Y cell. J Appl Toxicol 30: 649-655, 2010

26. Wang H, Chanas B and Ghanayem BI: Cytochrome P450 2E1 (CYP2E1) is essential for acrylonitrile metabolism to cyanide: comparative studies using CYP2E1-null and wild-type mice. Drug Metab Dispos 30: 911-917, 2002.

27. Gunasekar PG, Sun PW, Kanthasamy AG, Borowitz JL and Isom GE: Cyanide-induced neurotoxicity involves nitric oxide and reactive oxygen species generation after $\mathrm{N}$-methyl-D-aspartate receptor activation. J Pharmacol Exp Ther 277: 150-155, 1996.

28. Li L, Prabhakaran K, Shou Y, Borowitz JL and Isom GE: Oxidative stress and cyclooxygenase-2 induction mediate cyanide-induced apoptosis of cortical cells. Toxicol Appl Pharmacol 185: 55-63, 2002.

29. Esmat A,El-Demerdash E, El-Mesallamy H and Abdel-Naim AB: Toxicity and oxidative stress of acrylonitrile in rat primary glial cells: preventive effects of $\mathrm{N}$-acetylcysteine. Toxicol Lett 171: 111-118, 2007. 